\title{
Searching for HI Absorbing Gas in AGN
}

\author{
Y.M. Pihlström \& J.E. Conway \\ Onsala Space Observatory, S-439 92 Onsala, Sweden \\ R.C. Vermeulen \\ Netherlands Foundation for Research in Astronomy, P.O. Box 2, 7990 \\ AA Dwingeloo, The Netherlands
}

\begin{abstract}
With the aim of probing HI gas in AGN we have searched different samples of radio sources for the $21 \mathrm{~cm}$ atomic hydrogen line in absorption. Depending on the source morphology, we are able to probe gas that either belongs to the host galaxy ISM, or gas that is situated on scales $<1 \mathrm{kpc}$ which then may be connected with the AGN fueling. For the HI absorption detected in $3 \mathrm{C} 216$, we argue that such sub-kpc scale gas instead might be due to a jet-cloud interaction.
\end{abstract}

\section{Introduction}

The energy output from AGN is thought to be the result from accretion onto a super-massive object, powered by the infall of gas transported from the host galaxy down to the central regions. In fact, central structures of gas, usually with radii $<1 \mathrm{kpc}$, are frequently observed with the HST in nearby FRI radio galaxies (Verdoes Kleijn et al. 1999). These dust disks are also reported in molecular and atomic lines, e.g. in NGC4261 (van Langevelde et al. 2000).

While these central gas structures more or less are directly linked to the AGN activity, a related question is where the gas in these system originated? Since we expect that AGN luminosity is accretion powered, it is not obvious why the most powerful radio sources reside in elliptical hosts which are often assumed to be gas poor. Lately however, the traditional view of ellipticals as very gas poor is changing. It has been found that some early-type galaxies can contain significant amount of both molecular (see e.g. Knapp \& Rupen 1996) and atomic (Oosterloo, Morganti \& Sadler 1999) gas which could act as a fuel reservoir for the central engine.

In order to probe HI gas on these two scales we have, using the WSRT UHF receivers, performed HI absorption observations of three different types of radio sources (see Sect. 2).

\section{CSOs - probes of ISM gas and sub-kpc scale gas}

Compact Symmetric Objects (CSOs) and Compact Steep Spectrum sources (CSSs) are small $(<1 \mathrm{kpc})$ double lobe sources with random orientation, which 
probe both circumnuclear gas and the host ISM. We detected 3/14 CSO/CSSs in absorption, with an average column density $\mathrm{N}_{H I}=1.3 \times 10^{20}\left(T_{s p} / 100 K\right) \mathrm{cm}^{-2}$.

In particular, in the CSS $3 \mathrm{C} 216$ a $280 \mathrm{~km} / \mathrm{s}$ wide absorption line of opacity 0.003 was observed (Pihlström et al. 1999). This source also displays blazar characteristics, which in addition to superluminal motion in the mas jet indicates an end-on orientation. Given that the host of $3 \mathrm{C} 216$ is elliptical the estimated HI mass would exceed $3 \times 10^{9} \mathrm{M}_{\odot}$ if the $\mathrm{HI}$ is distributed over the whole galaxy, therefore we find it unlikely the detected absorption is due to normal ISM gas. It is also unlikely that the absorption would be due to a central torus, since the end-on orientation would require a very thick structure in order to cause the observed HI column. In addition, X-ray observations imply much lower total column densities towards the core than what we measure. One possible explanation to the absorption is that it arises in a jet-cloud interaction, in a region where the jet bends $0.85 \mathrm{kpc}$ from the core. EVN VLBI observations have been performed to determine the location of the absorbing gas.

\subsection{FRIIs - circumnuclear gas against their cores}

Central $(<1 \mathrm{kpc})$ gas can also be probed by selecting FRII radio galaxies, which have their orientation in the plane of the sky, and look for absorption towards their cores. Successful observations of five FRIIs at WSRT resulted in one detection (in 3C234), but this absorption was seen against one of the lobes. However, upper limits of the HI column density against the core in $3 \mathrm{C} 234$, as well as of the other sources in our sample, show that there still might be similar HI column densities as in the FRII prototype Cyg A (Conway 1999).

\subsection{Radio lobes as probes of ISM gas}

In order to test the possibility of using radio lobes as background probes for the ISM of the host galaxies, we selected a sample of end-on quasars and radio galaxies. Unified schemes predict the quasars to be end-on and thus this orientation would allow detections of gas against the far lobe. We detected 0 of 14 these quasars/radio galaxies, and assuming a typical HI extent of $10 \mathrm{kpc}$ our upper limits corresponds to $\mathrm{M}_{H I} \leq 2.8 \times 10^{8} \mathrm{M}_{\odot}$. This is consistent with the host galaxies being normal ellipticals with low HI content.

\section{References}

Conway, J.E, 1999, in ASP Conf. Ser. Vol. 156, Highly Redshifted Radio Lines, ed. C. Carilli, S. Radford, K. Menten \& G. Langston, 259

Knapp, G.R., \& Rupen, M.P. 1996, ApJ, 460, 271

van Langevelde, H.J., Pihlström, Y.M., Conway, J.E., Jaffe, W., \& Schilizzi, R.T. 2000, A\&A, 354, L45

Oosterloo, T.A., Morganti, R., \& Sadler, E. 1999, PASA, 16, 28

Pihlström, Y.M., Vermeulen, R.C., Taylor, G.B., \& Conway, J.E. 1999, ApJ, $525, \mathrm{~L} 13$

Verdoes Kleijn, G.A., Baum S.A., de Zeeuw, P.T., \& O'Dea C.P. 1999, AJ, 118, 2592 